\title{
Cognitive Structures of Good and Poor Novice Problem Solvers in Physics
}

\author{
Ton de Jong \\ Department of Philosophy and Social Sciences \\ Eindhoven University of Technology, \\ Eindhoven, The Netherlands
}

\author{
Monica G. M. Ferguson-Hessler \\ Department of Physics \\ Eindhoven University of Technology, \\ Eindhoven, The Netherlands
}

\begin{abstract}
The way knowledge is organized in memory is generally expected to relate to the degree of success in problem solving. In the present study, we investigated whether good novice problem solvers have their knowledge arranged around problem types to a greater extent than poor problem solvers have. In the subject of physics (electricity and magnetism), 12 problem types were distinguished according to their underlying physics principles. For each problem type, a set of elements of knowledge containing characteristics of the problem situation, declarative knowledge, and procedural knowledge was constnucted. All of the resulting 65 elements were printed on cards, and first-year university students in physics $(N$ $=47$ ) were asked to sort these cards into coherent piles shortly after they had taken an examination on electricity and magnetism. Essentially, good novice problem solvers sorted the cards according to problem types; the sorting by the poor problem solvers seemed to be determined to a greater extent by the surface characteristics of the elements. We concluded than an organization of knowledge around problem types might be highly conducive to good performance in problem solving by novice problem solvers.
\end{abstract}

In literature about problem solving in semantically rich domains, two research trends can be distinguished. One trend for research pays attention mainly to the process of solution (Reif, Larkin, \& Brackett, 1976; Schoenfeld, 1979). The other trend focuses on the knowledge of the problem solver and, particularly, on the organization of the knowledge (Chi, Feltovich, \& Glaser, 1981; Chi, Glaser, \& Rees, 1982; Larkin, 1979). A shift of interest from general, more or less domain-indcpendent problem-solving strategies to the domain-related knowledge base of the problem solver can be seen (Greeno, 1980).

First of all, problem solving in semantically rich domains requires knowledge of the subject matter involved. A series of interesting experiments by Perfetto, Bransford, \& Franks (1983), however, showed that the mere presence of knowledge in memory does not necessarily imply that this knowledge will be available at the time of problem solving. This availability of knowledge can be enhanced by an adequate organization of knowledge in memory, in other words, by a suitable cognitive structure; therefore, such a structure can play a crucial role in problem solving.

According to Chi et al. (1982), a cognitive structure adcquate for problem solving is composed of problem schemata. By a problem schema, they mean a set of elements of knowledge that are closely linked with each other within

We are grateful to Wim Vaags, Don Mellenbergh, and Christiaan Hamaker for their very helpful comments throughout the course of the experiment.

Correspondence concerning this article should be addressed to Ton de Jong, Department of Philosophy and Social Sciences, Eindhoven University of Technology, P.O. Box 513, $5600 \mathrm{MB}$ Eindhoven, The Netherlands. the knowledge base of the problem solver and that concern a particular type of problem. Problem types can be considered at several levels (Mayer, 1981). Here problem types are separated from each other according to the subject-matter principle or combination of principles that have to be applied to solve them.

Chi et al. (1981) asked experts and novices to categorize physics problems. Their results indicated that experts tended to sort problems according to the underlying physics principle (deep structure), whereas novices attended to the surface characteristics of the problem situations. From this and other studies, they inferred that experts' knowledge bases are organized differently from novices: Experts possess more adequate and complete problem schemata. This is consistent with the findings of a study by Larkin (1979). She measured time intervals between the principles generated by an expert and those by a novice while solving problems of physics. The expert generated principles in clusters (chunks), whereas the novice generated principles randomly in time.

The content of an adequate problem schema in memory is not restricted to solution principles. In addition to declarative knowledge (principles, formulae, and concepts), a problem schema should also contain characteristics of problem situations so that a connection between an actual problem and the problem schema is possible (Schoenfeld \& Herrmann, 1982). Moreover, an adequate problem schema should contain procedural knowledge, knowledge about actions that are necessary for solving that particular type of problem (Braune \& Foshay, 1983). In addition to these three kinds of schema-specific knowledge, the problem solver also needs strategic knowledge. This is the knowledge that tells the problem solver the stages that he or she has 
to follow in the problem-solving process (e.g., making a plan before working out). Schoenfeld (1979) called these managerial strategies. Mostly, the strategies are rather general, which implies that their applicability is not restricted to one type of problem. Therefore, this kind of knowledge does not have to be implied in specific problem schemata.

A knowledge base that is organized according to problem schemata allows the expert confronted with a problem to quickly select the correct declarative and procedural knowledge from memory. It does seem, however, that not only experts but also novices possess problem schemata. Silver (1979), who asked novice problem solvers in mathematics to sort mathematical problems, discovered the same sort of differences between good and poor problem solvers that $\mathrm{Chi}$ ct al. (1981) and Chi et al. (1982) found between experts and novices. A conclusion from the Silver study could, thus, be that differences in cognitive structure also exist between good and poor novice problem solvers.

Studies involving the cognitive structures of novices have been conducted in several domains, such as mathematics (Shavelson \& Stanton, 1975), psychology (Fenker, 1975), physics (Thro, 1978) and geology (Champagne, Klopfer, Desena, \& Squires, 1981). The methods used in these studies are more direct than the catagorization of problems that was used by Silver (1979). In all these studies, subjects were asked to interrelate concepts selected from the domain. (In the subject-matter field of electricity and magnetism, these concepts could be flux or magnetic field, for example.) The way in which these relations were made differed between the studies. Shavelson and Stanton discussed three of those methods, word association, card sorting, and graph building. In word association, for example, the degree of relatedness of two concepts for a subject is calculated on the basis of the number and rank order of common associations given by the subject on both concepts. A common result of these studies is that during instruction, relations between concepts given by novices change and become similar to the relations given by experts. In this way, the novices' cognitive structure becomes more similar to the cognitive structure for experts. Surprisingly, a positive correlation between this degree of similarity and the ability of novices to solve problems could sometimes (Fenker; Thro) but not always be demonstrated (Geeslin \& Shavelson, 1975; Shavelson, 1973).

This last result seems to contradict the conclusions of the Chi et al. (1981) and the Chi et al. (1982) studies. When experts have their knowledge organized according to problem schemata and this benefits the solving of problems, a clear correlation should be expected between the degree of similarity of expert and novice cognitive structure and the problem solving abilities of the novices. This contradiction, however, is on the surface. The aforementioned more direct studies of the cognitive structure paid attention only to the organization in memory of one kind of knowledge, namely, concepts. The other kinds of knowledge that constitute adequate problem schemata (other parts of declarative knowledge, characteristics of problem situations, and procedural knowledge) were not considered. Therefore, from the data of these studies, it cannot be determined whether cognitive structures are made up of problem schemata.

The present study was designed primarily to test the hypothesis that good novice problem solvers have their knowledge organized according to problem schemata, as opposed to poor novice problem solvers, who were expected to lack this kind of organization. Along the lines of the more direct methods for measuring the cognitive structure (Shavelson \& Stanton, 1975), we developed an experimental task that required subjects to determine relations between elements of knowledge from a physics topic, electricity and magnetism. In the set of elements, characteristics of problem situations, declarative knowledge, and procedural knowledge were included. In this way, the results of the measurement could be expected to reveal problem schemata occurring in the cognitive structure.

The experimental task was carried out by a group of firstyear university students shortly after they had taken an examination on the subject of electricity and magnetism. The results of the examination were used as a measure of their problem-solving ability in that specific domain.

A second question concerned the cognitive structure of experts. As mentioned before, Chi et al. (1982) concluded that experts' knowledge is built up of problem schemata. Reif and Heller (1982) took a somewhat different position. They suggested that the knowledge of experts is hierarchically organized. This means that their knowledge is arranged on different levels of detail: The higher levels give very general (and mostly rather abstract) laws and definitions, which are worked out and specified at the lower levels. To gain some insight into the cognitive structures of experts, we asked a group of four expert physicists to carry out the same experimental task as the novices.

\section{Method}

\section{Instructional Materials}

The subject of electricity and magnetism was taught to the participants of this experiment as part of the regular study program. It covered the following topics: static electric and magnetic fields, the Kirchhoff laws, the Lorenz force, and electromagnetic induction. The course consisted of three lectures a week during the first 9 weeks of the academic year, supplemented by $2 \mathrm{hr}$ of instruction in problem solving weekly in groups of approximately 25 students. Lecture notes and a collection of exercises and former examination problems were used by the students.

\section{Examination}

The course concluded with an examination consisting of five open problems, divided into two to five subproblems covering most of the topics already mentioned. In these problems, a physical system was described (e.g., a capacitor), and in each of the subproblems, students were asked to find an unknown quantity (e.g., the charge on one of the conductors of the capacitor). The examination papers were graded on a 10-point linear scale, where $6(60 \%$ score) was a passing mark and 10 was excellent. The experimenters were not involved in giving the course or in constructing or grading the examinations. 


\section{Subjects}

Of a group of 98 first-year University of Technology students that took the examination for electricity and magnetism, $47 \mathrm{com}$ plied with a request to participate in the experiment. They were not paid and received no credit for their participation. The mean score on the examination for the complete group of students was $5.0(S D=1.89)$ and for the participants in the experiment, 5.4 $(S D=1.84)$. The four experts were staff members involved in teaching about electricity and magnetism, and all had many years of experience teaching this subject.

\section{Experimental Materials}

The subject matter of electricity and magnetism was divided into six main themes (e.g., electrostatics and magnetostatics). In doing so, the laws of Kirchhoff were excluded because they form a separate unit with little relation to the rest of the subject. Within the six main themes, 12 types of problems were distinguished. Each type was related to a fundamental principle (e.g., Coulomb's law).

Elements of knowledge were then constructed around each problem type so that every problem type consisted of at least 1 clcment of declarative and 1 of procedural knowledge needed to solve that type of problem as well as at least 1 characteristic of a problem situation where the principle is useful. Most problem types consisted of more than the minimum of 3 elements. For the 12 problem types, a total of 65 elements resulted. Of course, this list of elements was not exhaustive for the subject matter. No overlap of elements between problem types existed.

The second author, a physicist with many years of teaching experience, defined the problem schemata and constructed the knowledge elements, using previous examinations and textbook exercises as sources. Our colleagues responsible for teaching and examining the participants in the experiment were not involved.

The 65 elements of knowledge are listed in the Appendix. To identify them, we labeled each element successively with a numeral (one of the six main themes of the subject matter), a letter (problem type within the subject-matter theme), and another numeral (element of knowledge within the problem type). Thus, the 12 problem types can be recognized by the combination of the tirst numeral and the letter. They can be characterized by the following physics principles: Coulomb's law (1A), Gauss's law (1B), Biot-Savart's law (2A), Ampère's law (2B), magnetic dipole field (2C), capacitor (3A), method of images (3B), potential (4A), charged particle in a combined electric and magnetic field (5A), force on a current $(6 \mathrm{~A})$, electromotive force (emf) induced in a moving conductor (6B), and current induced in a circuit $(6 \mathrm{C})$. Characteristics of problem situations are represented by the first one or two knowledge elements of each problem type, and procedural knowledge is contained in the last or last two elements of knowledge of each problem type (1A5, 1B5, 1B6, 2A3, 2A4, 2B5, 2B6, 2C4, 2C5, 3A7, 3B5, 3B6, 4A6, 5A4, 5A5, 6A5, $6 \mathrm{~B} 3,6 \mathrm{C} 6$, and 6C7). Declarative knowledge is represented in the remaining elements. As an example, Problem Type 6B, which concerns the electromagnetic force induced in a moving conductor, consists of three elements of knowledge. The first (6B1) gives characteristics of a problem situation, "the emf induced in a straight conductor rotating in a magnetic field." The second element (6B2) gives declarative knowledge, in this case a formula, $U=v \cdot B \cdot l$, and the third element of knowledge (6B3) gives a procedure, "adding the emf induced in various elements." In the Appendix, it is indicated for every element of knowledge whether it concerns characteristics of problem situations (CofP), declarative knowledge (DK), or procedural knowledge (PK).

To illustrate the different kinds of elements of knowledge, we will elaborate on the following very simple problem concerning Coulomb's law (Problem Type 1A): Two point charges, $+q$ and $-q$, have coordinates $(-a, 0)$ and $(+a, 0)$, respectively. Calculate the electric field intensity in point $P$, situated at $(-b, 0)$. (See Figure 1.)

The characteristics of this problem situation (given two point charges, calculate the electric field) form a special case of Element 1A1. The solution of this problem runs along the following lines: Each of the two point charges gives rise to an electric field intensity at $P$. The magnitude and direction of these vectors are given by Coulomb's law (1A4) in combination with the definition of the electric field intensity (1A2). The principle of superposition (1A3) states that the total field is found by superposing the fields of individual charge elements. In addition to these three elements of declarative knowledge, the problem solver has to calculate the field at $P$ for each of the two point charges and add the vectors to find the total field. This procedure is given in Element $1 \mathrm{A5}$.

An analysis of the examination that the subjects of this experiment took revealed that one of its five problems included applying the Kirchhoff laws, which were part of the subject matter excluded from the 12 problem types. All the subproblems of the remaining four problems could be classified into the 12 problem types or, in a few cases, as a combination of two of them. Altogether, 8 of the 12 problem types were represented in the examination.

\section{Procedure}

One of the methods discussed by Shavelson and Stanton (1975) for cliciting knowledge of relations between concepts was the sorting of cards. This method does not require complicated instruction, and it has been used when the number of concepts was substantial (Burton, 1972; Miller, 1969; Rapoport \& Fillenbaum, 1972). Therefore, this method was considered suitable for this experiment.

Each of the 65 elements was printed on a small card, and each participant was given a deck of these cards in random order. In written instructions, they were told to construct coherent piles. They had to sort the cards so that cards sorted together in their opinion were more closely connected to each other than to cards in other piles. No criteria for this coherence were suggested to them. In the instructions, they were told that the number of piles made and the number of cards in each pile were up to them. The participants were asked to check their piles again after their first sorting to see if they could combine or split some of their piles. Cards depicting elements of knowledge with which the students were unfamiliar had to be kept separate and indicated as such.

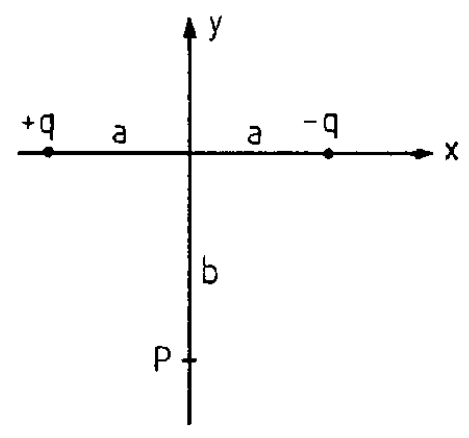

Figure 1. Two point charges in a Cartesian coordinate system. 
They were asked to label their piles after they had completed the sorting task, indicating the reason for their placing the elements together in a particular pile. The four experts received the same instructions.

\section{Scoring}

Each subject's sorting was converted into a $65 \times 65$ symmetrical matrix, with each cell of the matrix representing a particular pair of elements of knowledge. The cell was numbered one when both elements were placed in the same pile and zero when they were placed in separate piles. (The matrices being symmetrical, from now on only the part above the diagonal will be considered.)

This procedure was also followed for an artificial sorting of the cards into 12 piles according to the 12 problem types that had been constructed in advance. This resulted in a $65 \times 65$ symmetrical matrix, the upper half of which contained 151 cells numbered one and 1,929 cells numbered zero. This "ideal" sorting was called problem-type centered, and the resulting ideal matrix was called the problem-type-centered, or PC, matrix.

For every student, the number of times a one in a particular cell of the matrix for this student coincided with a one in the PC matrix (the $S_{1,1}$ score) was added and also the number of times a one in the matrix coincided with a zero in the PC matrix (the $S_{1,0}$ score for this student). A measure of the problem-type centeredness of the sorting by the student was then calculated according to Equation 1.

$$
\mathrm{PC}=\frac{S_{1,1}}{151}-\frac{S_{1,0}}{1,929}
$$

This measure had a PC value of 1 when the student sorted the cards into the 12 problem types. The PC value was 0 when only one pile was made of all 65 elements. The PC score could be negative, having a minimum value of -.16 . This valuc was reached when the elements were sorted so that no elements of the same problem type were sorted into the same pile $\left(S_{1,1}=0\right)$ and, moreover, so that the number of piles made was as small as possible in that condition. A negative value of $P C$ indicated a sorting that was extremely different from a problem-type-centered sorting.

\section{Results}

Two kinds of analysis were made with the data of the novices. First, a correlation coefficient was calculated between the PC scores and scores in the examination for the whole sample, and second, a cluster analysis (Johnson, 1967) was applied to the summed matrices of a group of good problem solvers (score on the examination, or $x, \geq 7, n=$ 13 ) and to the summed matrices of a group of poor problem solvers $(x \leq 3, n=7)$. (Actually, these students failed their exams. Students with a score of 1 , the lowest possible score, were excluded from the sample). The middle range of the sample was excluded here to minimize the chance that students were placed in the wrong group due to inadcquacies in grading. The two summed matrices were formed by summing the values within cells over subjects.

The PC scorcs for the subjects ranged between -.067 and $.878(M=.347, S D=.162)$. The correlation between examination scores and PC scores was $.40(p<.01)$. There was only one student with a negative PC score. When we analyzed his sorting more closely, it appeared that his sorting was different from that of all other participants. This student made a small number of piles with a large number of cards in each pile. The piles were based on what can be called a "functional" characteristic of the elements. For example, he grouped together formulae that could be deduced. When this student, who was a good problem solver $(x=8)$, was removed from the analysis, the correlation between examination scores and PC scores rose to $.54(p$ $<.01)$.

The results of a cluster analysis (maximum method; Johnson, 1967) applied to the summed matrices of the group of good $(x \geq 7)$ and the group of poor $(x \leq 3)$ problem solvers are presented in Figures 2 and 3, respectively. It is useful to bear in mind when interpreting these figures that 12 separate clusters should result from an ideal, problemtype-centered cognitive structure, each containing the elements of knowledge of one of the 12 problem types exclusively.

The results of the cluster analysis illustrate that the sorting by the group of good problem solvers was essentially problem-type centered, with clusters of elements being composed of the various problem types $(1 \mathrm{~A}, 1 \mathrm{~B}, 2 \mathrm{~A}$, and so forth). When the separate clusters in Figure 2 are regarded as piles of cards and a PC score is calculated then, this PC score has a value of .64 .

In Figure 2, it can be seen that elements of the separate problem types cluster together. The first cluster, for example, is mainly made up of Problem Types IA, 3B, and $4 \mathrm{~A}$. Within this relatively large cluster, the strongest connections occur between the elements of each problem type, however. In two other clusters, problem types belonging to one of the main themes of the subject matter are clustered $(6 \mathrm{~A}, 6 \mathrm{~B}$, and $6 \mathrm{C}$ and $2 \mathrm{~A}$ and $2 \mathrm{C})$.

The clusters for the group of poor problem solvers (Figure 3) illustrate that their sorting can certainly not be called problem-type centered. (The PC score for the cluster analysis was .22.) The sorting by the poor problem solvers seemed to be determined to a substantial extent by surface characteristics of the elements. For example, five out of the seven elements of the cluster $2 \mathrm{~A} 1,2 \mathrm{~A} 2,2 \mathrm{C} 2,2 \mathrm{C} 3,6 \mathrm{~A} 4$, $2 \mathrm{~B} 2$, and $2 \mathrm{~B} 3$ contain one or both of the symbols $B$ and $m$. Other clusters can be characterized by words, such as fields or calculate, or by a combination of words and a symbol, such as energy and work and $w$. On the other hand, a little insight into the subject matter might be present regarding the cluster that is made up of elements of Problem Types $6 \mathrm{~B}$ and $6 \mathrm{C}$ exclusively.

The results of the sorting tasks of the experts were more difficult to interpret than those of the students. The PC scores for their sortings were low or moderate, having values of $.09, .20, .40$, and .41 . A further analysis of their sorting revealed that one of them used a functional approach, one like that used by the student with the negative PC score. This expert gave his piles labels such as electrostatic formulae and mathematical techniques. In the sorting of the other three experts, hierarchical characteristics could be identified. Also, this was indicated by the labels they used, for example, theory of electric field and applications of electric field. Additionally, two of these three experts had a (functional) pile labeled mathematics. 
$1 \mathrm{~A} 1$

$1 \mathrm{~A}$

$1 \mathrm{~A} 5$

$1 \mathrm{A3}$

$1 \mathrm{~A} 4$

$3 \mathrm{~A} 5$

3A 7

3B3

$4 \mathrm{~A} 1$

$4 \mathrm{~A} 3$

$4 \mathrm{~A} 2$

$4 \mathrm{~A} 5$

3B1

उB 6

3B5

3B4

1BI

$1 \mathrm{~B} 2$

1B3

1B5

$1 \mathrm{~B} 4$

$6 \mathrm{C} 3$

2A1

2B1

$2 \mathrm{~A} 3$

$2 \mathrm{~A} 4$

$2 \mathrm{C} 4$

$2 \mathrm{C} 2$

$2 \mathrm{C} 3$

$6 \mathrm{~A} 4$

$2 \mathrm{C} 5$

B2

2B3

2B4

$3 A 1$

$3 A 2$

$3 \mathrm{~A} 3$

$3 \mathrm{~A}$

$5 A 1$

$5 \mathrm{~A}$

$5 \mathrm{~A} 5$

$6 \mathrm{~A} 2$

$5 \mathrm{~A} 3$

$6 \mathrm{~A}$

$6 \mathrm{~A} 3$

$6 \mathrm{~B} 1$

$6 \mathrm{~B} 2$

$6 \mathrm{CI}$

$6 \mathrm{C} 3$

$6 \mathrm{C} 5$

$6 \mathrm{C} 4$

$6 \mathrm{C} 2$

$6 \mathrm{~A} 5$

$6 \mathrm{~B} 3$
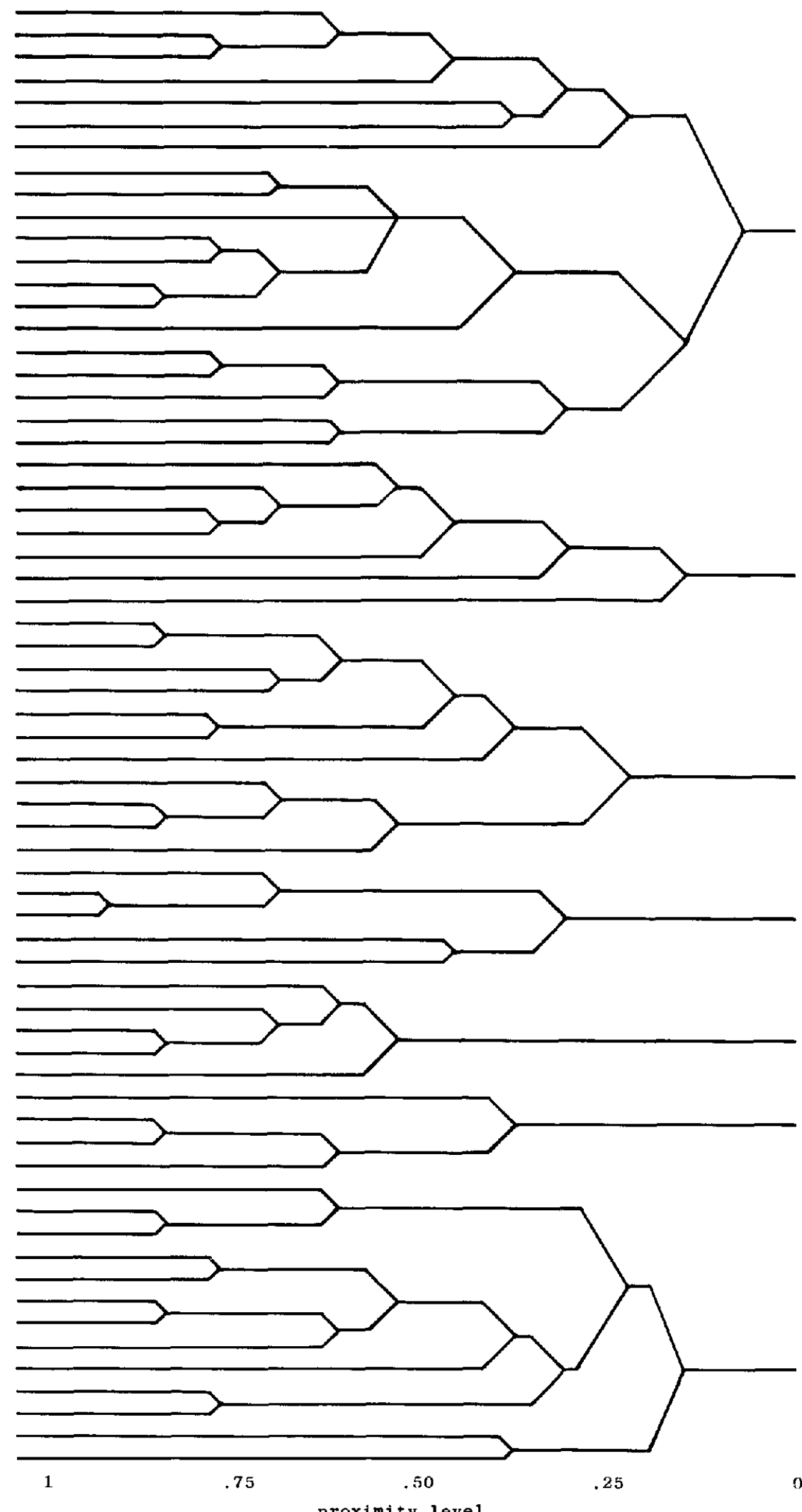

Figure 2. Hierarchical cluster analysis for the group of students with $x \geq 7(n=13)$. 


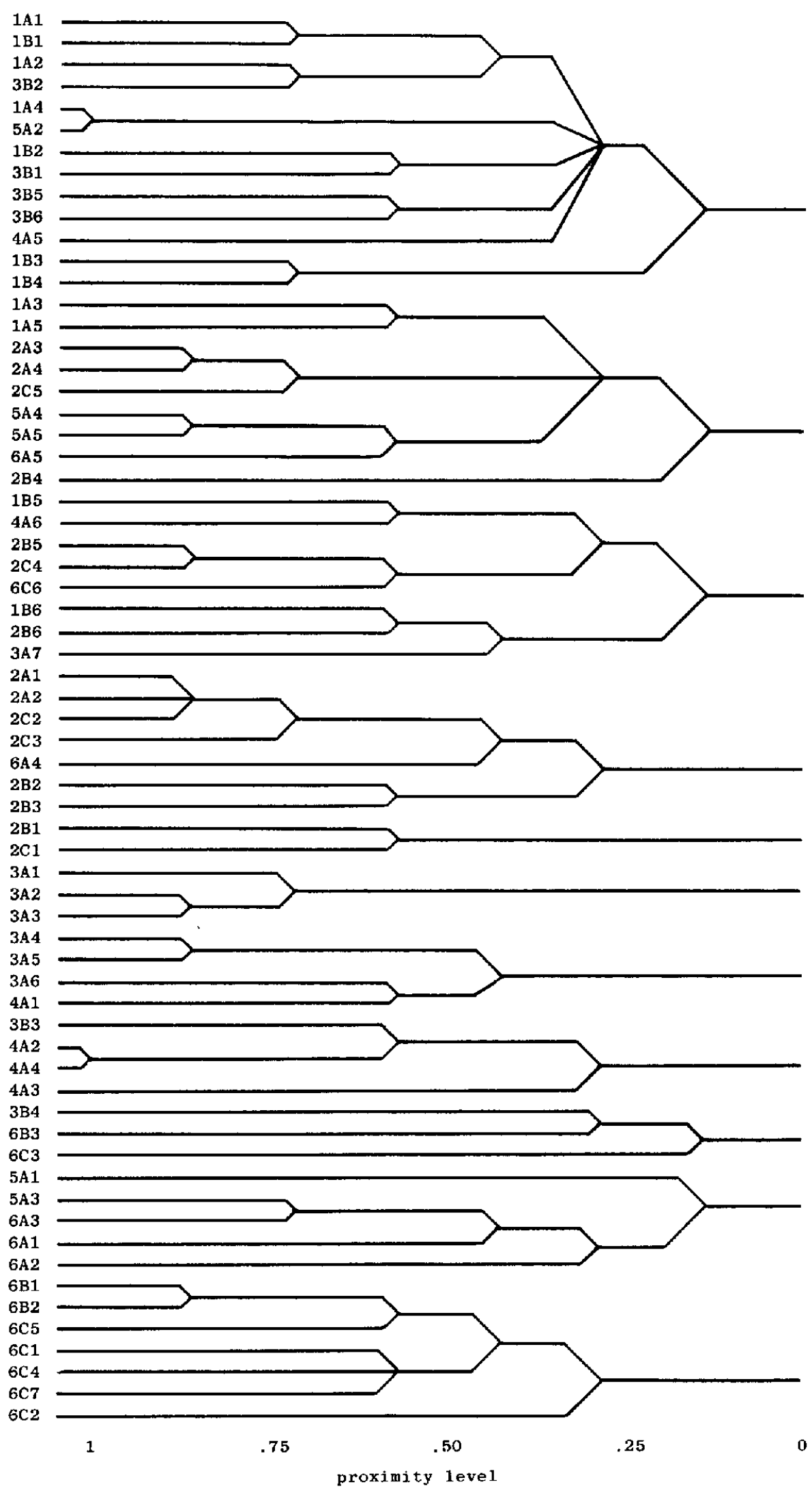

Figure 3. Hierarchical cluster analysis for the group of students with $x \leq 3(n=7)$. 


\section{Discussion}

The results of the present study support the hypothesis that good novice problem solvers have their knowledge organized in a more problem-type-centered way than poor ones. The correlation found between exam scores and PC scores can be regarded as quite high when considering all the factors besides knowledge organization that can possibly influence the degree of success in problem solving, such as miscalculations and emotional and motivational factors.

One restriction should be applied to these conclusions. This experiment gave only correlational data, from which a causal relation between cognitive structure and success in problem solving cannot be deduced. Nevertheless, the results indicate the existence of such a relation.

A cognitive structure made up of adequate problem schemata is a very efficient knowledge base for solving problems and, thus, for getting high grades on examinations. Once a student recognizes the relevant characteristics in the description of the problem, the declarative and procedural knowledge needed for the solution become available, and the problem can be solved in a straightforward way (assuming that the student has a basic knowledge of physics and mathematics).

It could be argued that the poor students simply did not prepare for the exam and, therefore, were unfamiliar with the instructional material and that this explains their results in the exam and the sorting task. This did not, however, seem to be the case. In the sorting task, students were asked to make separate piles of single cards when they were unfamiliar with the element on the card. The mean number of unfamiliar cards was very low for the complete group of students ( $u=1.4)$, and the mean number for the group of poor students $(u=2.6)$ was not much higher than for the group of good students $(u=0.8)$. This outcome indicates that not the amount but the organization of knowledge is related to problem-solving ability.

The low PC scores of the experts are not really surprising. The knowledge of electricity and magnetism included in the course is only part of the expert's total knowledge of classical electromagnetism. This knowledge includes a number of laws and concepts that are more general and abstract than the laws and concepts taught to first-year students. Such knowledge is difficult to harbor in a set of problem schemata and is probably arranged in a more or less hierarchical way (as proposed by Reif \& Heller, 1982) in the memory of an expert. A hierarchical structure can result in elements of knowledge that are related to each other at a higher level in the structure being sorted together, although they belong to different problem schemata. For example, one of the experts had a pile labeled theory of electric field, which contained 10 elements of knowledge belonging to five problem types. In a hierarchical-structure, however, 8 of these elements fit in the same place, namely, the specification of the concept field. Also, the grouping of elements of knowledge that play a certain (functional) role in the problem-solving process (the mathematical techniques) undoubtedly lowered the PC scores for the experts. For students, these techniques are related to certain types of problems. Experts, with their very elaborate knowledge, can use those techniques in many places and, probably for that reason, have not bound it to specific problem schemata. Obviously, however, the elements of knowledge used in this study do not provide enough information for definite conclusions on the existence of hierarchical structures.

Hierarchical and problem-type-centered cognitive structures are not mutually exclusive. Problem types (characteristics of problem situations) can be coupled to the relevant laws and formulae at the lowest level of a hierarchical structure. This level then turns out to be built up from more or less complete problem schemata. Within such a structure, the principles of solving different types of problems are easily deduced. This can be regarded as a somewhat different explanation for the results of the experts in the studies of Chi et al. (1981) and Chi et al. (1982). The participants in the present study were not given any criterion for sorting together the elements of knowledge. Experts can be expected to construct the 12 problem types from the 65 elements of knowledge when explicitly asked.

Recently, the importance of assessing the cognitive structures of novices was stressed by Diekhoff (1983). In the present study, we argued that in this assessment, restrictions to concepts of the subject matter should not be advocated. Organization in memory of characteristics of problem situations, declarative knowledge, and procedural knowledge should be the main point of interest, in both instruction and assessment. Reif (1984) advocated the teaching of hierarchical structures to novices. He acknowledged, however, that an adequate cognitive structure for novices could be different from an adequate structure for experts. A hierarchical structure of knowledge in memory that seems to exist for a number of experts may not be within the reach of novices because they lack the comprehensive knowledge needed. In that case, the present study suggests that a problem-type-centered cognitive structure can be a useful interim stage for novices on their way to expertise.

\section{References}

Braune, R., \& Foshay, W. R. (1983). Towards a practical model of cognitive information processing task analysis and schema acquisition for complex problem-solving situations. Instructional Science, 12, 121-145.

Burton, M. (1972). Semantic dimensions of occupation names. In A. K. Romney, R. N. Shepard, \& S. B. Nerlove (Eds.), Multidimensional scaling (pp. 55-73). New York: Seminar Press. Champagne, A. B., Klopfer, L. E., Desena, A. T., \& Squires, D. A. (1981). Structural representation of student's knowledge before and after science instruction. Journal of Research in Science Teaching, 18, 97-111.

Chi, M. T., Feltovich, P. J., \& Glaser, R. (1981). Categorization and representation of physics problems by experts and novices. Cognitive Science, 5, 121-152.

Chi, M. T., Glaser, R., \& Rees, E. (1982). Expertise in problem solving. In R. J. Sternberg (Ed.), Advances in the psychology of human intelligence (pp. 7-77). Hillsdale, NJ: Erlbaum.

Diekhoff, G. M. (1983). Testing through relationship judgments. Journal of Educational Psychology, 75, 227-233.

Fenker, R. M. (1975). The organization of conceptual materials: 
A methodology for measuring ideal and actual cognitive structures. Instructional Science, 4, 33-57.

Geeslin, W. E., \& Shavelson, R. J. (1975). An exploratory analysis of the representation of a mathematical structure in students' cognitive structures. American Educational Journal, 12, 22-29.

Greeno, J. G. (1980). Trends in the theory of knowledge for problem solving. In D. T. Tuma \& F. Reif (Eds.), Problem solving and education (pp. 9-25). Hillsdale, NJ: Erlbaum.

Johnson, S. C. (1967). Hierarchical clustering schemes. Psychometrica, 32, 241-254.

Larkin, J. H. (1979). Processing information for effective problem solving. Engineering Education, 285-288.

Mayer, R. E. (1981). Frequency norms and structural analysis of algebra story problems into families, categories and templates. Instructional Science, 10, 135-175.

Miller, G. A. (1969). A psychological method to investigate verbal concepts. Journal of Mathematical Psychology, 6, 169-191.

Perfetto, G. A., Bransford, J. D., \& Franks, J. J. (1983). Constraints on access in a problem solving context. Memory and Cognition, $11,24-31$.

Rapoport, A., \& Fillenbaum, S. (1972). An experimental study of semantic structures. In A. K. Romney, R. N. Shepard, \& S. B. Nerlove (Eds.), Multidimensional scaling (pp. 55-73). New York: Seminar Press.

Reif, F. (1984). Understanding and teaching problem solving in physics. Research on physics education: Proceedings of the first international workshop Lalonde les Maures (pp. 15-53). Paris: Centre National du Recherche Scientifique.

Reif, F., \& Heller, J. I. (1982). Knowledge structures and problem solving in physics. Educational Psychologist, 17, 102-127.

Reif, F., Larkin, J. H., \& Brackett, G. C. (1976). Teaching general learning and problem solving skills. American Journal of Physics, 44, 212-217.

Schoenfeld, A. H. (1979). Can heuristics be taught? In J. Lochhead \& J. Clement (Eds.), Cognitive process instruction (pp. 315-338). Philadelphia, PA: Franklin Institute Press.

Schoenfeld, A. H., \& Herrmann, D. J. (1982). Problem perception and knowledge structure in expert and novice mathematical problem solvers. Journal of Experimental Psychology: Learning. Memory, and Cognition, 8, 484-494.

Shavelson, R. J. (1973). Learning from physics instruction. Journal of Research in Science Teaching, 10, 101-111.

Shavelson, R. J., \& Stanton, G. C. (1975). Construct validation: Methodology and application to three measures of cognitive structure. Journal of Educational Measurement, 12(2), 67-85.

Silver, E. A. (1979). Student perceptions of relatedness among mathematical verbal problems. Journal for Research in Mathematics Education, 10, 195-210.

Thro, M. P. (1978). Relationships between associative and content structure of physics concepts. Journal of Educational PSychology, 70, 971-978.

Appendix

\section{Problem Type IA}

1A1 (CofP):

The field of a semi-infinite straight line carrying a charge

1 A3 (DK):

The principle of superposition

1A5 (PK):

Calculating the vector sum of the contributions to the elec-

tric field of various elements
IA2 (DK):

The definition of electric field intensity

$1 \mathrm{~A} 4$ (DK):

$$
\mathrm{d} \mathbf{F}=\frac{Q \mathbf{e}}{4 \pi \epsilon_{0} r^{2}} \mathrm{~d} q
$$

\section{Problem Type $1 B$}

1BI (CofP):

The field of a charge density with cylindrical symmetry

$1 B 3$ (DK):

Electric flux

1B5 (PK):

Choosing a surface on which $E_{n}$ is constant

\section{Problem Type $2 A$}

$2 \mathrm{Al}$ (CofP):

The field of a semi-infinite straight wire, carrying a current

\section{$2 \mathrm{~A} 3(\mathrm{PK})$ :}

Establishing the possible directions in which the magnetic field of a current-carrying wire may have components
$1 \mathrm{~B} 2(\mathrm{DK})$ :

Charge enclosed

1B4 (DK):

Closed surface

1B6: (PK)

Calculating the volume integral $\iiint \rho(r) \mathrm{d} V$

2A2 (DK):

$$
\mathrm{dB}=\frac{\mu_{0} \mathrm{I}(\mathrm{dl} \times \mathbf{e})}{4 \pi r^{2}}
$$

$2 \mathrm{~A} 4$ (PK):

Adding the various contributions to the magnetic induction in a chosen direction

\section{Problem Type $2 \mathrm{~B}$}

2B1 (CofP):

The field of a current density $\mathrm{J}(r)$ with cylindrical symmetry
2B2 (DK):

Current enclosed 
2B3 (DK):

The line integral of $\mathbf{B}$ around a closed path

2B5 (PK):

Choosing a closed path, on which $B_{s}$ is constant or zero
2B4 (DK):

The right-hand screw rule for line integrals around a closed path

2B6 (PK):

Deciding on the direction in which to calculate a line integral around a closed path

\section{Problem Type 2C}

$2 \mathrm{C} 1$ (CofP):

The field at great distance from an arbitrary circuit

$2 \mathrm{C} 3(\mathrm{DK}):$

$$
|\mathbf{m}|=I A
$$

2C5 (PK):

Calculating the vector sum of a number of magnetic moments

\section{Problem Type $3 \mathrm{~A}$}

3A1 (CofP):

Two thin conducting coaxial cylinders, one of which is free to move along the axis

3A3 (DK):

The definition of capacity

3 A5 (DK):

$$
W=1 / 2 \epsilon_{0} E^{2}
$$

3A7 (PK):

Calculating the line integral $\int \mathbf{E} \cdot \mathrm{ds}$ from the surface of one conductor to the surface of another conductor

Problem Type $3 B$

3BI (CofP):

$3 \mathrm{~B} 2$ (DK):

A grounded spherical conductor in the field of a point charge

3B3 (DK):

Equipotential surface with $V=0$

3B5 (PK):

Introducing an imaginary charge into the system

\section{Problem Type $4 A$}

4A1 (CofP):

Work required in order to place a point charge at a given point in an electrical field

4A3 (DK):

$$
P \int^{\infty} \mathbf{E} \cdot \mathrm{d} \mathbf{s}
$$

4A5 (DK):

$$
\oint \mathbf{E} \cdot \mathrm{d} \mathbf{s}=0
$$

5A1 (CofP):

The motion of a charged particle in combined electric and magnetic fields

3A6 (DK):

\section{Problem Type 5A}

$5 \mathrm{~A} 2(\mathrm{DK})$ :
$3 \mathrm{~A} 2$ (CofP):

The mutual forces on the two conductors of a capacitor

3A4 (DK):

Increase of ficld energy $=$ external work + energy delivered by voltage source
$E=0$ inside a conductor

3B4 (DK):

3B6 (PK):

Calculating the charge density that is induced on a grounded conductor
4A2 (DK):

The definition of potential

4A4 (DK):

$V(\mathbf{r})$ is continuous everywhere

4A6 (PK):

Choosing a path along which the line integral of the electric field intensity can be calculated in a simple way

$$
\mathbf{F}=q \mathbf{E}
$$


$5 A 3(\mathrm{DK}):$

$$
\mathbf{F}=q(\mathbf{v} \times \mathbf{B})
$$

5A5 (PK):

Separately analyzing the motion of a particle in different directions

\section{Problem Type 6A}

$6 \mathrm{~A} 1$ (CofP):

The acceleration of a wire loop carrying a current, moving freely in a magnetic field

6A3 (DK):

$$
\mathbf{F}=l(\mathbf{I} \times \mathbf{B})
$$

6A5 (PK):

Calculating the vector sum of the forces acting on various elements of the current

\section{Problem Type 6B}

6B1 (CofP):

The electromotive force induced in a straight conductor rotating in a magnetic field

6B3 (PK):

Adding the electromotive force induced in various elements

\section{Problem Type 6C}

$6 \mathrm{Cl}$ (CofP):

The current in a wire loop moving in a magnetic field

6C3 (DK):

Faraday's law

$6 \mathrm{C} 5$ (DK):

$$
\phi=L \cdot I
$$

$6 \mathrm{C} 7(\mathrm{PK})$ :

Establishing the direction of an induced current
5A4 (PK):

Resolving the force on a particle into components parallel and perpendicular to the velocity
6A2 (DK):

$6 \mathrm{~A} 4(\mathrm{DK}):$

$$
\begin{aligned}
\mathbf{F} & =m \mathbf{a}_{\mathrm{M}} \\
M & =\text { center of mass }
\end{aligned}
$$

6B2 (DK):

$$
\mathbf{M}=\mathbf{m} \times \mathbf{B}
$$

6C2 (DK):

Magnetic flux

6C4 (DK):

Lenz's law

$6 \mathrm{C6}(\mathrm{PK})$ :

Calculating the surface integral $\iint(B \cdot n) d A$ 